\title{
Evolution non-linéaire de la houle dans la zone de shoaling
}

Françoise Becq

Etudiante en thèse, EDF - Laboratoire National d'Hydraulique, 6. quai Watier 78400 Chatou

Michel Benoit

Ingénieur - chercheur, EDF - Laboratoire National d'Hydraulique, 6, quai Watier 78400 Chatou

Philippe Forget

Chercheur CNRS, L.S.E.E.T, Université de Toulon et du Var BP $132 \quad 83957$ La Garde

Résumé

L'étude porte sur le phénomène d'interactions non-linéaires entre triplets de fréquences qui survient dans le domaine côtier. Des mesures expérimentales réalisées au Laboratoire National d'Hydraulique ont permis de mieux appréhender le processus physique. La comparaison de ces mesures à des résultats de simulations numériques montre qu'il est important de prendre en compte les effets des nonlinéarités qui agissent tant sur la répartition spectrale de l'énergie que sur les moments du spectre (hauteur significative, période moyenne, ...). Le phénomène étant plus prononcé en eau très peu profonde, un cas-test avec déferlement est également présenté.

\section{Introduction}

La connaissance fine des conditions d'environnement est un préalable obligatoire pour la conception des ouvrages côtiers et la prédiction de la dynarnique sédimentaire. Or, les conditions hydrodynamiques liées à la houle se trouvent fortement modifiées en faible profondeur, sous l'action combinée de multiples processus (réfraction, shoaling, déferlement, effets non-linéaires, ...). Cette étude a pour objectif d'appréhender plus particulièrement le phénomène d'interactions nonlinéaires entre trois ondes, qui survient dans la zone de shoaling et induit de profondes modifications sur le champ de vagues.

\section{Présentation du phénomène}

En eau très peu profonde $(k h<<1)$, les vagues sont essentiellement non dispersives. Des interactions résonnantes entre plusieurs ondes peuvent alors survenir dès le second ordre dans le cadre de la théorie faiblement non-linéaire : ce sont les interactions entre triplets de fréquences où deux composantes satisfaisant à la relation de dispersion linéaire forcent le mouvement d'une troisième onde dont la fréquence et le nombre d'onde sont imposés par ceux des deux ondes primaires: 


$$
\begin{aligned}
& \mathrm{f}_{1} \pm \mathrm{f}_{2}=\mathrm{f}_{3} \\
& \overrightarrow{\mathrm{k}}_{1} \pm \overrightarrow{\mathrm{k}}_{2}=\overrightarrow{\mathrm{k}}_{3}
\end{aligned}
$$

En milieu faiblement dispersif, Armstrong et al. (1962) ont montré qu'un échange significatif d'énergie peut également avoir lieu entre les trois composantes. L'onde 3, dont la phase et la vitesse de propagation sont imposées par celles des ondes 1 et 2 , ne peut plus obéir exactement à la relation de dispersion; on dit alors que c'est une onde liée. La théorie des ondes liées suppose qu'un transfert univoque d'énergie survient pour générer des harmoniques supérieures ou inférieures; en réalité un retour d'énergie vers les fréquences primaires a lieu, conduisant à des interactions "presque résonnantes » (Madsen et Sorensen, 1993). Les effets se caractérisent par une distorsion des profils de vagues. D'autre part, le spectre d'énergie se trouve fortement modifié par l'apparition de nouveaux pics spectraux.

\section{Modélisation des interactions entre triplets de fréquences}

\subsection{Présentation du modèle}

Les équations de Boussinesq sont les plus couramment utilisées pour modéliser l'évolution d'un champ de vagues en eau peu profonde car elles permettent d'inclure les effets d'une faible dispersion et d'une faible non-linéarité sous les hypothèses que $\mu^{2}=(\mathrm{kh})^{2} \ll 1, \varepsilon=(\mathrm{a} / \mathrm{h}) \ll 1$ et $O(\varepsilon)=O\left(\mu^{2}\right)$ où $\mathrm{k}$, h, et a représentent respectivement le nombre d'onde, la profondeur d'eau et l'amplitude des vagues. La restriction majeure de ce type d'équations est leur limitation de profondeur d'eau. C'est pourquoi Madsen et Sorensen (1992) ont proposé une nouvelle forme des équations de Boussinesq, où l'introduction d'une constante d'ajustement $B$ permet d'améliorer les propriétés de dispersion linéaire et les mécanismes de shoaling associés au modèle. Les équations de Boussinesq étendues sont transformées dans le domaine spectral par décomposition en séries de Fourier de l'élévation de la surface libre et de la vitesse intégrée sur le fond. Madsen et Sorensen (1993) obtiennent ainsi une équation d'évolution pour les amplitudes de Fourier complexes, valable pour des vagues unidirectionnelles se propageant sur des fonds en pentes douces, qui s'écrit :

$$
\frac{\mathrm{dA}_{\mathrm{p}}}{\mathrm{dx}}=-\mathrm{C}_{\text {shoal }} \frac{\mathrm{h}_{\mathrm{x}}}{\mathrm{h}} \mathrm{A}_{\mathrm{p}}-2 \mathrm{ig}\left[\mathrm{F}_{\mathrm{p}}^{+}+\mathrm{F}_{\mathrm{p}}^{-}\right]
$$

Le premier terme du membre de droite représente le coefficient de shoaling qui dépend de la profondeur relative $(\mathrm{kh})$ et du paramètre de dispersion $\mathrm{B}$. Dans le cas où $B=1 / 15$, Madsen et Sorensen (1993) ont montré que ce terme correspondait bien au coefficient de shoaling linéaire basé sur la théorie de Stokes du premier ordre. La valeur classique $\mathrm{B}=0$, qui permet de retrouver les équations de Boussinesq standards, conduit au contraire à une sous-estimation importante du terme de shoaling. Le second terme de droite modélise les interactions non-linéaires entre triplets de fréquences au travers de $\mathrm{F}_{\mathrm{p}}^{+}$et $\mathrm{F}_{\mathrm{p}}^{-}$qui traduisent, respectivement, les transferts d'énergie vers les harmoniques supérieures et inférieures. L'importance des transferts d'énergie est gouvernée par les relations de phases entre les composantes formant le triplet et par les coefficients d'interaction issus de transformation spectrale de la partie non-linéaire des équations de Boussinesq. 


\subsection{Inclusion de la dissipation par déferlement dû aux fonds}

Eldeberky et Battjes (1996) proposent d'étendre le modèle à la zone de déferlement par l'inclusion d'un terme de dissipation. Ils supposent que le déferlement n'est pas affecté par les interactions non-linéaires entre triplets de fréquences et utilisent le modèle paramétrique de Battjes et Janssen (1978), qui exprime la variation du flux d'énergie totale le long d'un profil bathymétrique. Le terme de dissipation totale dû au déferlement $\left(\mathrm{D}_{\mathrm{tot}}^{\mathrm{BJ}}\right)$ doit être réparti selon les fréquences. Le modèle suppose le déferlement indépendant de la fréquence, ce qui conduit au terme de dissipation sur les amplitudes de Fourier, ajouté directement au membre de droite de (2):

(3) $\mathrm{d}_{\mathrm{br}}=-\frac{1}{2} \frac{\mathrm{D}_{\mathrm{tot}}^{\mathrm{BJ}}}{\mathrm{F}_{\mathrm{tot}}} \mathrm{A}_{\mathrm{p}} \quad$, où $\mathrm{F}_{\mathrm{tot}}$ représente le flux total d'énergie.

\subsection{Mise en oeuvre numérique}

L'équation d'évolution pour les amplitudes de Fourier complexes est intégrée grâce à la méthode de Runge Kutta d'ordre 4, ce qui permet le calcul de l'élévation de la surface libre ou des spectres d'énergie le long d'un profil bathymétrique.

Pour des applications où seul le spectre de houle aléatoire est connu comme condition à la limite, Eldeberky et Battjes (1996) proposent de discrétiser ce spectre en bandes de fréquences $(\Delta f)$ et de supposer que $\left|A_{p}\right|=\left(1 / 2 E_{p} \Delta f\right)^{1 / 2}$. Les phases initiales de chaque composante p sont tirées aléatoirement. Pour éliminer l'influence des phases initiales, une moyenne sur 20 réalisations successives est faite, ainsi qu'une moyenne glissante sur trois points des spectres obtenus.

\section{Comparaison des résultats de simulations numériques à des mesures en laboratoire}

\subsection{Description des mesures}

Des séries de mesures ont été réalisées aù Laboratoire National d'Hydraulique afin de mieux appréhender le phénomène d'interactions non-linéaires entre triplets de fréquences. Les expériences ont été effectuées dans ün carial de $45 \mathrm{~m}$ de long et $0.60 \mathrm{~m}$ de large, équipé d'un générateur de houle aléatoire. Un profil de plage schématique a été construit, le long duquel 16 sondes ont été placées pour permettre l'enregistrement temporel de l'élévation de la surface libre (figure 1). Les analyses statistiques et spectrales réalisées sur les données enregistrées pour chaque sonde ont fourni une description qualitative et quantitative de l'évolution des trains d'ondes.

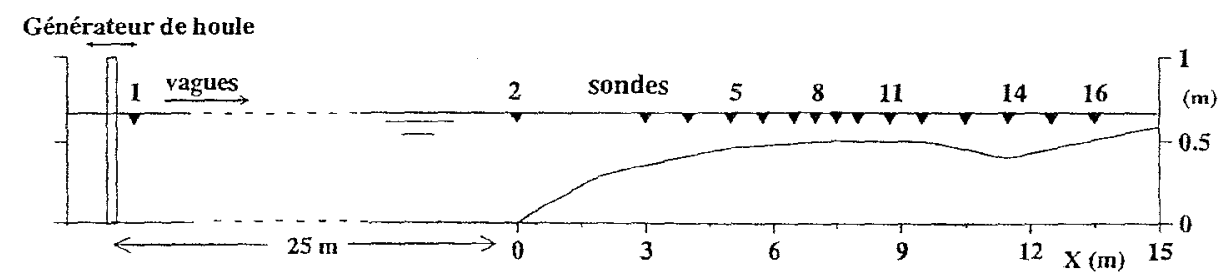

Figure 1. : Description du dispositif expérimental. 


\subsection{Présentation d'un cas-test sans déferlement}

\subsubsection{Description du cas-test}

Le spectre imposé au niveau du générateur de houle est un spectre de type JONSWAP ayant une fréquence de pic de $0.4 \mathrm{~Hz}$ et une hauteur significative relativement faible, de $3.3 \mathrm{~cm}$, pour éviter le déferlement des vagues. Le spectre mesuré au niveau de la sonde 2 a été utilisé comme condition initiale pour les simulations numériques. La profondeur d'eau au niveau du générateur est de $0.65 \mathrm{~m}$.

\subsubsection{Comparaison des simulations numériques aux mesures}

Les mesures expérimentales ont été comparées à des résultats de simulations obtenūes avec un modèle linéaire classique, puis avec le modèle déterministe de type Boussinesq. L'évolution des spectres de variance mesurés le long du profil bathymétrique (figure 2) montre qu'un transfert d'énergie important vers les hautes fréquences survient dans la partie peu profonde du canal. Ce transfert d'énergie conduit à la génération de nouveaux pics spectraux aux harmoniques de la fréquence de pic. Les mauvais résultats issus du modèle linéaire montrent bien que ce transfert d'énergie est induit par des effets non-linéaires qui ne peuvent pas être négligés. La figure 2 fait apparaitre également que le modèle de type Boussinesq est capable de reproduire correctement l'influence des interactions non-linéaires qui gouvernent les transferts d'énergie au sein du spectre. Les spectres calculés s'apparentent convenablement aux spectres mesurés, et ce jusqu'au niveau de la plage (sonde 16). Les figures 3.a et 3.b représentent respectivement l'évolution des hauteurs significatives spectrales (HM0) et des périodes moyennes (TM01), le long du profil bathymétrique. La comparaison entre les mesures et les résultats du modèle linéaire montre que les non-linéarités ont un effet moins marqué sur les hauteurs de vagues puisque le phénomène est conservatif en énergie (Komen et al., 1994), mais qu'elles induisent des écarts significatifs de la période moyenne avec les estimations de la théorie linéaire. Le modèle de Boussinesq améliore la prévision des hauteurs significatives et donne une représentation convenable de l'évolution des périodes moyennes le long du profil, même si ces dernières sont légèrement surestimées.

\subsection{Présentation d'un cas-test avec déferlement}

\subsubsection{Description du cas-test}

Le spectre imposé au niveau du générateur est un spectre de type JONSWAP ayant une fréquence de pic de $0.435 \mathrm{~Hz}$ et une hauteur significative de $8.0 \mathrm{~cm}$. La profondeur d'eau a été abaissée à $0.61 \mathrm{~m}$ au niveau du générateur pour permettre un déferlement important des vagues.

\subsubsection{Comparaison des simulations numériques aux mesures}

L'analyse des spectres de variance mesurés (figure 4) montre d'une part, 

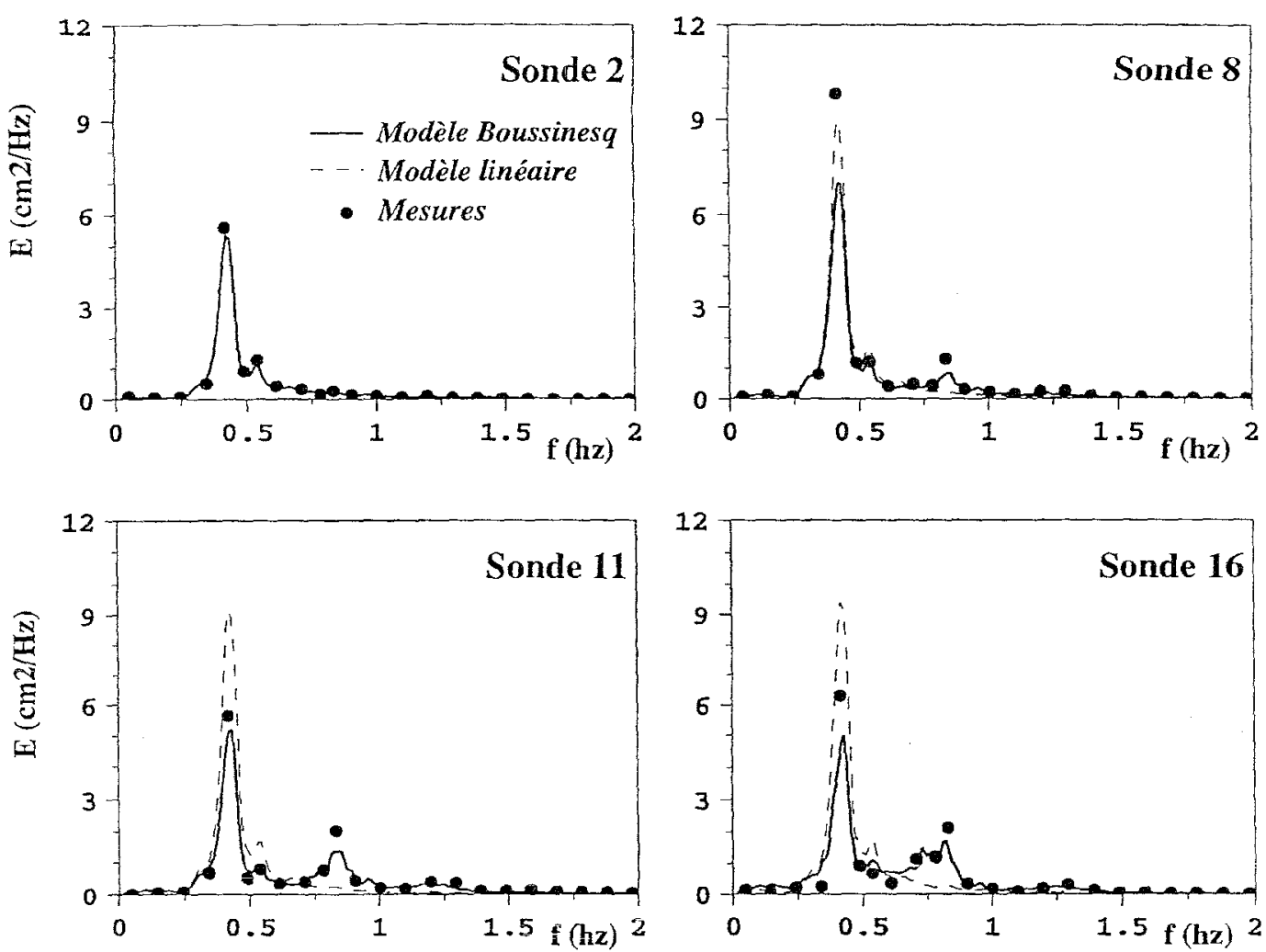

Figure 2: : Evolution des spectres de variance le long du profil bathymétrique.
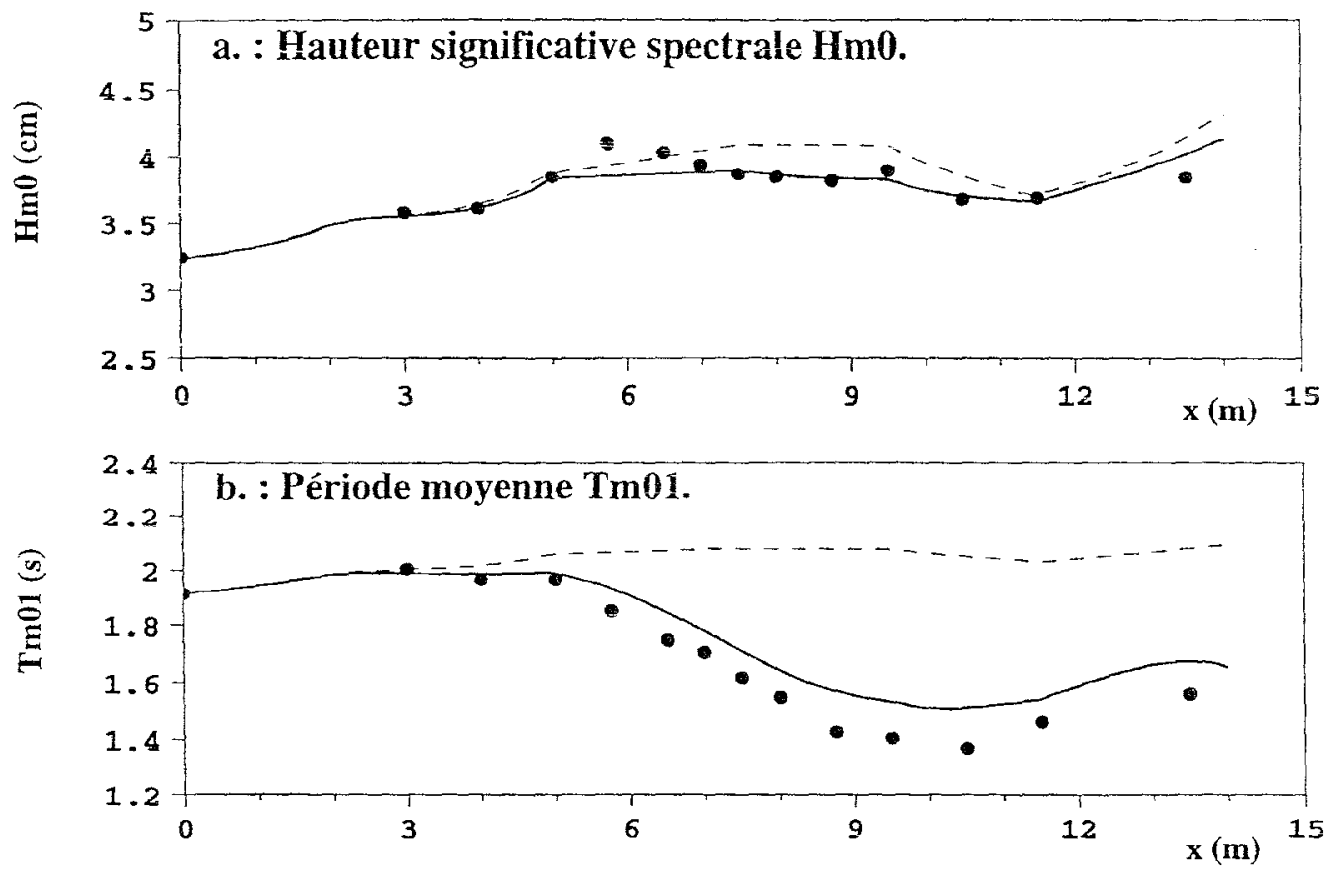

Figure 3. : Evolution de $\mathrm{Hm0}$ et $\mathbf{T m 0 1}$ le long du profil bathymétrique.

Les mesures $(\bullet)$ sont comparées à un modèle linéaire $(--)$, puis au modèle de Boussinesq (-). 

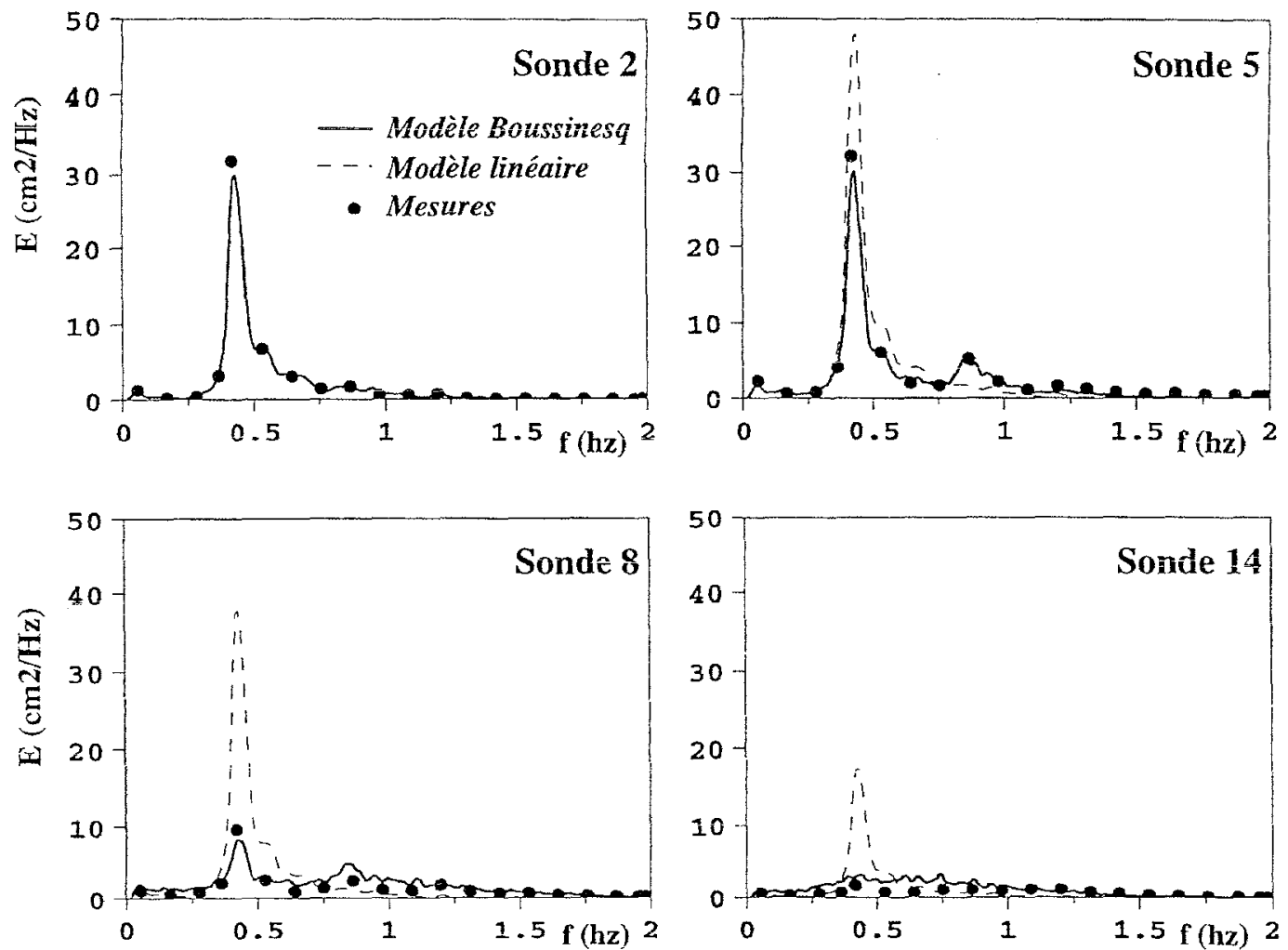

Figure 4. : Evolution des spectres de variance le long du profil bathymétrique.
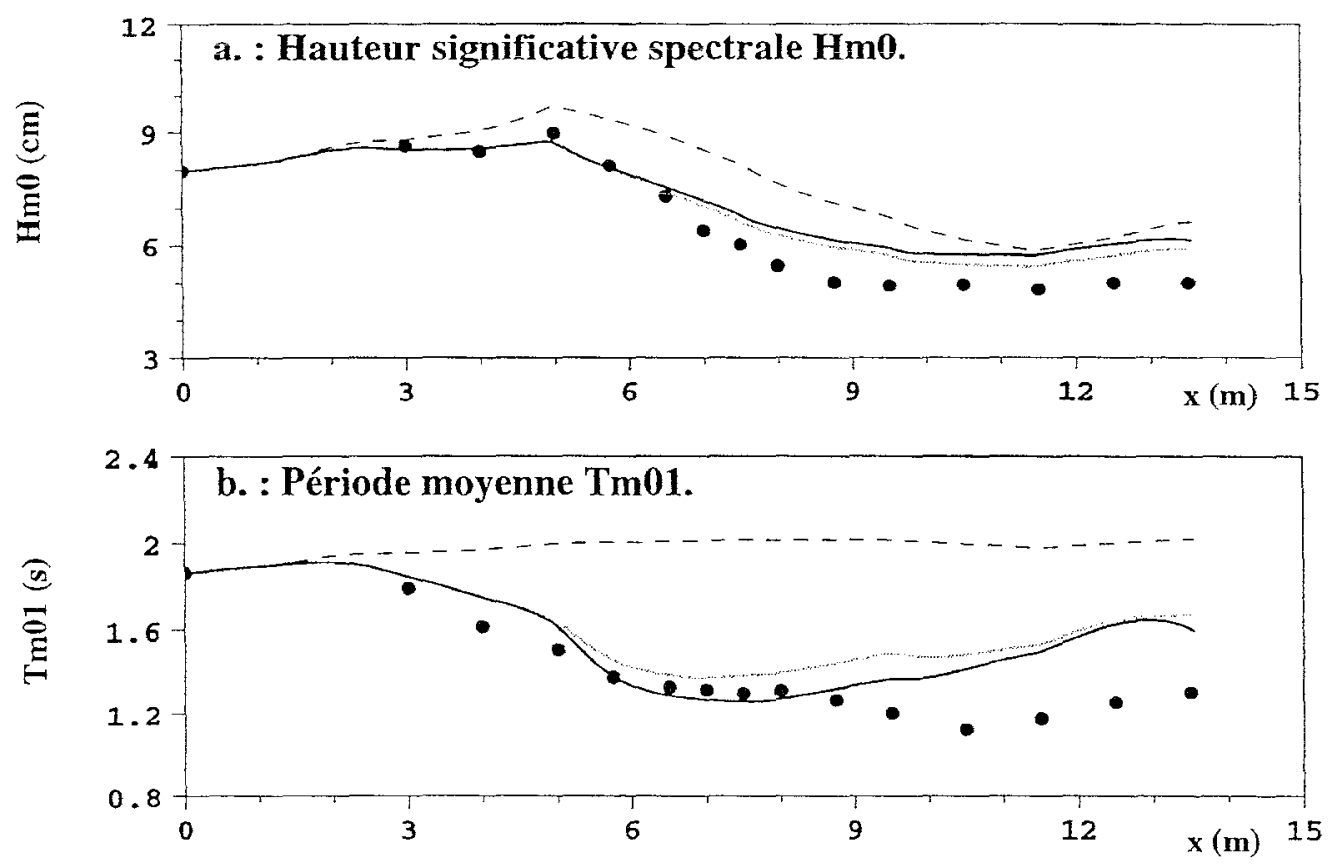

Figure 5. : Evolution de $\mathrm{Hm0}$ et $\mathrm{Tm01}$ le long du profil bathymétrique.

Les mesures $(\bullet)$ sont comparées à un modèle linéaire $(\cdot-\bullet)$, puis au modèle de Boussinesq en supposant un déferlement indépendant de la fréquence $(-)$ ou en $\mathrm{f}^{2}(\ldots \ldots)$. 
l'apparition précoce d'interactions non-linéaires entre triplets de fréquences (sonde 5) et, d'autre part, un déferlement important dans la zone peu profonde, qui conduit à une très forte réduction de l'énergie contenue dans le spectre incident. Les mesures sont comparées à des résultats de simulation obtenus avec un modèle linéaire, puis avec le modèle de Boussinesq. Les deux modèles intègrent un terme de dissipation par déferlement dû au fond, mais seul le second permet de reproduire correctement l'évolution des spectres de variance le long du profil bathymétrique.

L'analyse des paramètres synthétiques (figure 5) montre que le modèle de Boussinesq simule bien l'évolution qualitative de la hauteur significative spectrale malgré une légère surestimation des valeurs mesurées. Certains auteurs (Mase et Kirby (1992), Chen et Guza (1997)) préconisent l'utilisation d'un terme de dissipation par déferlement composé de deux membres dont le premier est indépendant de la fréquence et le second varie comme le carré de la fréquence. Un coefficient de pondération permet de répartir plus ou moins l'énergie sur chacun des deux membres. En supposant une dissipation par déferlement entièrement proportionnelle à la fréquence au carré (courbe en grisé), on aboutit à une faible amélioration du calcul de HMo.

Le calcul de la période moyenne par le modèle de Boussinesq (figure 5.b) permet de bien reproduire les mesures jusqu'à la sonde 11. Au delà de cette sonde, on note une surestimation de la période moyenne. La prise en compte du phénomène d'interactions non-linéaires améliore considérablement les résultats comparativement à l'utilisation d'un modèle linéaire. Il faut noter également qu'en conséquence du déferlement très important qui survient sur le haut fond, le spectre de variance se trouve très atténué et relativement plat (sonde 14 de la figure 4). Cela rend d'autant plus difficile l'évaluation de la période moyenne. La prise en compte d'un terme de dissipation par déferlement fonction de la fréquence au carré n'améliore pas les résultats obtenus et conduit à des valeurs un peu supérieures aux précédentes.

\section{Conclusions}

L'étude en canal de l'évolution spectrale d'un champ de vagues le long d'un profil bathymétrique, montre que d'importants transferts d'énergie vers les harmoniques supérieures ont lieu dans la zone de shoaling. Ces transferts d'énergie résultent d'interactions non-linéaires entre triplets de fréquences. L'utilisation d'un modèle linéaire ne permet donc pas de reproduire les caractéristiques spectrales d'un champ de vagues dans la zone de shoaling et conduit à des erreurs significatives sur le calcul de période moyenne. Lors de la détermination des conditions hydrodynamiques liées à la houle en domaine côtier, il est important de prendre en compte ces non-linéarités. L'utilisation d'un modèle à résolution de phases, tel que celui proposé par Madsen et Sorensen (1993), permet de reproduire correctement l'évolution d'un champ de vagues dans la zone de shoaling et conduit à une bonne estimation des paramètres synthétiques (hauteur significative spectrale, période moyenne) en l'absence de déferlement. L'inclusion dans le modèle d'un terme de dissipation par déferlement dû aux fonds (Eldeberky et Battjes, 1996) permet d'étendre le domaine de validité et conduit à une bonne estimation de l'évolution spectrale du champ de vagues lors d'un déferlement important. 
Les modèles à résolution de phases sont les plus couramment utilisés pour reproduire l'évolution non-linéaire des vagues dans le domaine côtier. Toutefois le processus physique d'interaction entre triplets de fréquence peut également être simulé par des modèles à phases moyennées (Eldeberky et Battjes, 1995), ce qui permet de réduire le coût CPU.

Cette étude se limite à la propagation unidirectionnelle des vagues dans la zone de shoaling. Or, les effets directionnels peuvent être importants, les interactions entre triplets de fréquences pouvant conduire à la génération de nouveaux pics directionnels dans le cas de mers croisées (Elgar et al., 1993). Des développements sont en cours pour la prise en compte de ce phénomène dans le modèle spectral d'états de mer TOMAWAC développé au LNH (Benoit et al., 1996).

\section{Références}

ARMSTRONG J.A., BLOEMBERG N., DUCUING J. and PERSHAN P.S. (1962). Interaction between light waves in a nonlinear dielectric. Phys. Rev. B, 127: pp 1918-1939.

BATTJES J.A., JANSSEN P.A.E.M. (1978) : Energy loss and set-up due to breaking of random waves. Proc. ioth Int. Conf. on Coastal Eng., Vol 1, pp 569-587.

BENOIT M., MARCOS F., BECQ F. (1996) : Development of a third generation shallow-water wave model with unstructured spatial meshing. Proc. 25th Int. Conf. on Coastal Eng. (ASCE), pp 465-478.

CHEN Y.H. and GUZA R.T. (1997). Modeling spectra of breaking surface waves in shallow water. In press in J. Geophys. Res.

ELDEBERKY Y. and BATTJES J.A. (1995). Parameterisation of triads interactions in wave energy models. Proc. Int. Coastal Dynamics'95, pp 140-148.

ELDEBERKY Y., BATTJES J.A. (1996) : Spectral modelling of wave breaking : application to Boussinesq equations. J. Geophys. Res., 101 (C1), pp 1253-1264.

ELGAR S., GUZA R.T. and FREILICH M.H. (1993) : Observations of nonlinear interactions in directionally spread shoaling surface gravity waves. J. Geophys. Res., 98, pp 20299-20305.

KOMEN G.J., CAVALERI L., DONELAN M., HASSELMANN K. and HASSELMANN S. (1994) : Dynamics and modelling of ocean waves. Cambridge University Press.

MADSEN P.A. and SORENSEN O.R. (1992). A new form of the Boussinesq equations with improved linear dispersion characteristics. Part 2: a slowly-varying bathymetry. Coastal Eng., 18, pp 183-205.

MADSEN P.A. and SORENSEN O.R. (1993). Bound waves and triads interactions in shallow water. Ocean Eng., 20 (4), pp. 359-388.

MASE H. and KIRBY J.T. (1992). Hybrid frequency-domain KdV equation for random wave transformation. Proc. of the 19th Int. Conf. on Coastal Eng., pp 474-487. 